\section{EDITOR'S QUIZ: GI SNAPSHOT}

\section{Seeing a cause for blindness in severe pancreatitis}

During a holiday on a Mediterranean island, a 63-year old woman suffered a severe attack of pancreatitis secondary to gallstones. During the hospital stay, no sepsis was noted, but she was treated with prophylactic intravenous antibiotics, intravenous fluid and analgesia intensive care support was not required. After 22 days in hospital, she travelled back to the UK and presented to our department on advice to seek further help regarding ongoing symptoms of nausea and reduced appetite. A CT scan performed on admission demonstrated a developing $13 \times 4.5 \mathrm{~cm}$ acute pancreatic collection with fat necrosis, splenic vein occlusion and gastric compression. Treatment with analgesia, venous thrombosis prophylaxis and nasojejenual feeding was commenced pending either a possible regression or maturation of the collection when a drainage procedure would be performed. Repeated blood cultures performed for mild fever $\left(37.5^{\circ} \mathrm{C}\right)$ were negative. Twelve days later, a repeat CT scan showed persistence of the collection with a walled-off appearance and a coinciding rise in $\mathrm{C}$ reactive protein prompted transgastric endoscopic drainage. The following day urgent ophthalmology review was performed as the patient described that she had been seeing 'floaters' for 2 weeks but now was unable to read. Right eye visual acuity was $6 / 60$ and left eye was 6/12 (figure 1 ).

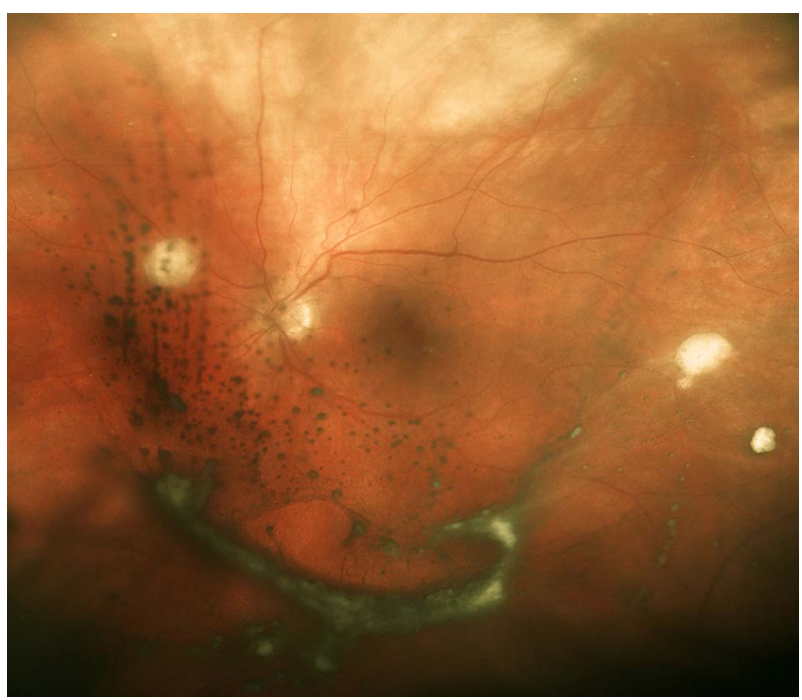

Figure 1 Opthalmology examination revealed bilateral intraocular inflammation, with fluffy white preretinal lesions and multiple 'string-of-pearls' signs.

\section{QUESTION}

What is the diagnosis? What risk factors does this woman have for developing this condition?

See page 46 for answer 


\section{EDITOR'S QUIZ: GI SNAPSHOT}

\section{Seeing a cause for blindness in severe pancreatitis}

\section{See page 32 for question}

\section{ANSWER}

These signs were diagnostic of a fungal endogenous endophthalmitis confirmed on a vitreous fluid culture of Candida albicans. This condition has a number of associations, including immunocompromise, prolonged intravenous access or drug abuse, and is a sight threatening disease. ${ }^{1}$ We believe this is the first case report of a fungal endophthalmitis secondary to translocation from an infected pancreatic fluid collection (PFC). The patient underwent intravitreal amphotericin injections, urgent bilateral vitrectomies, repeated endoscopic drainage and received 6 weeks oral voriconazole and intravenous anidulafungin ${ }^{2}$ (the latter added following endocarditis concerns) (figure 2).

There is a debateable suggestion that there is a strong correlation between fungal positivity in infected PFCs and use or duration of prophylactic antibiotics. ${ }^{3}$ Current UK guidelines for acute pancreatitis suggest prophylactic antibiotics should be considered only for patients with more than $30 \%$ necrosis, but the evidence to make any recommendation is conflicting. ${ }^{4}$ Initial conservative management avoiding drainage of non-infected PFCs is advised ${ }^{5}$ with guidelines recommending diagnostic aspiration or drainage if there are signs of infection. ${ }^{45}$ Given this further demonstration of the severe complications of pancreatitis, we would echo the current UK guideline recommendations of vigilance with prophylactic antibiotic treatment and a low threshold to perform early diagnostic aspiration.

\section{A D Hopper, ${ }^{1}$ D Gosling, ${ }^{2}$ S Patwary, ${ }^{2}$ D Partridge, ${ }^{3}$ M Peterson ${ }^{4}$ \\ ${ }^{1}$ Department of Gastroenterology, Sheffield Teaching Hospitals, Sheffield, UK ${ }^{2}$ Department of Ophthalmology, Sheffield Teaching Hospitals, Sheffield, UK ${ }^{3}$ Department of Microbiology, Sheffield Teaching Hospitals, Sheffield, UK ${ }^{4}$ Department of Surgery, Sheffield Teaching Hospitals, Sheffield, UK}

Correspondence to Dr Andrew D Hopper, Room J19, J Floor Department of Gastroenterology, Royal Hallamshire Hospital, Glossop Road, Sheffield S102JF, UK; andrew.hopper@sth.nhs.uk

Contributors ADH: drafting the work and conception or design of the work. DG, SP, DP and MP: drafting the work or revising it critically for important intellectual content. Final approval of the version published.

Competing interests None declared.

Patient consent Obtained.

Provenance and peer review Not commissioned; externally peer reviewed.

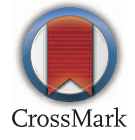

To cite Hopper AD, Gosling D, Patwary S, et al. Gut 2016;65:46.

Received 25 March 2015

Revised 4 April 2015

Accepted 6 April 2015

Gut 2016;65:46. doi:10.1136/gutjnl-2015-309662

\section{REFERENCES}

1 Vose M, Beatty S, Charles SJ. Candida endophthalmitis: an unusual complication of prolonged intravenous access. Postgrad Med J 2001;77:119-20.

2 Vaziri K, Schwartz SG, Kishor K, et al. Endophthalmitis: state of the art. Clin Ophthalmol 2015;9:95-108.

3 Trikudanathan G, Navaneethan U, Vege SS. Intra-abdominal fungal infections complicating acute pancreatitis: a review. Am J Gastroenterol 2011;106:1188-92.

4 Working Party of the British Society of Gastroenterology; Association of Surgeons of Great Britain and Ireland; Pancreatic Society of Great Britain and Ireland; Association of Upper GI Surgeons of Great Britain and Ireland. UK guidelines for the management of acute pancreatitis. Gut 2005;54(Suppl 3):1-9.

5 Da Costa DW, Boerma D, van Santvoort HC, et al. Staged multidisciplinary step-up management for necrotizing pancreatitis. Br J Surg 2014;101:e65-79.

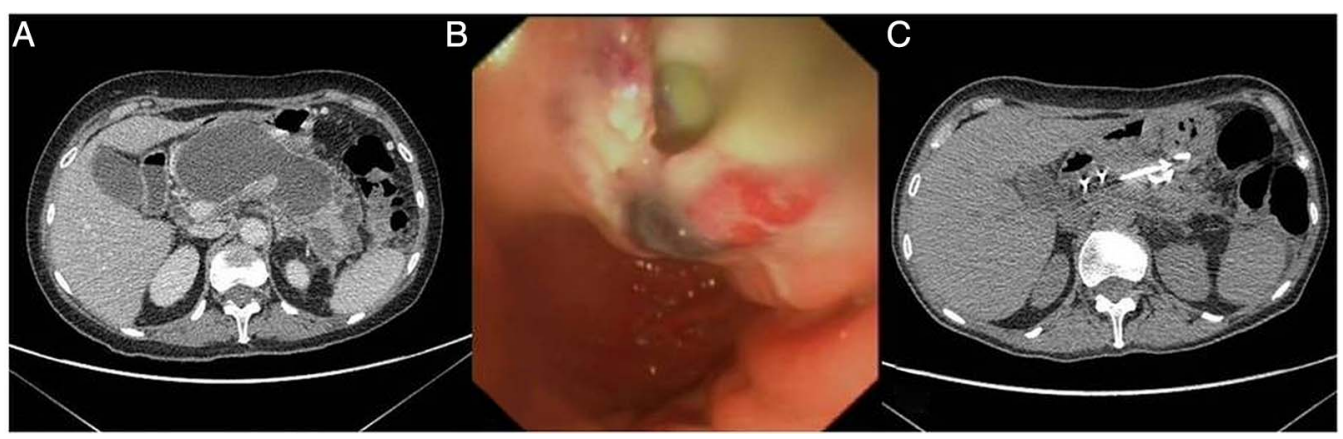

Figure 2 (A) CT of pancreatic collection prior to drainage. (B) Picture of endoscopic gastric drainage procedure with creation of wide cystgastrostomy (wire obscured by outflowing purulent fluid) with culture confirming Candida albicans. (C) Repeat CT 6 weeks after two further stent changes and washouts. The patient made a full recovery and went on to have a cholecystectomy. 